\title{
Conocimiento ancestral de plantas medicinales en la comunidad de Sahuangal, parroquia Pacto, Pichincha, Ecuador
}

\author{
Ancestral knowledge of medicinal plants in the community of Sahuangal, Pacto \\ parish, Pichincha, Ecuador \\ Conhecimento ancestral de plantas medicinais na comunidade de Sahuangal, \\ freguesia de Pacto, Pichincha, Ecuador
}

\author{
Luis Alberto Sarauz Guadalupe \\ luis.sarauz@cordillera.edu.ec \\ ORCID: 0000-0001-6211-8651
}

Instituto Tecnológico Superior Universitario Cordillera, Ecuador

Recibido 4 de noviembre 2020 | Arbitrado y aceptado 25 de noviembre 2020 | Publicado en 04 de enero 2021

\begin{abstract}
RESUMEN
Uno de los factores de gran importancia para la supervivencia y perpetuación de la humanidad ha sido el conocimiento y uso de plantas medicinales como medicamentos herbarios. En el presente estudio de caso se identificó los conocimientos ancestrales de los recursos botánicos de la parroquia Rural de Sahuangal, ubicada al noroccidente de Pichincha, Ecuador. La recolección de información se realizó a través de visitas técnicas, talleres participativos, entrevistas y encuestas a los pobladores de esta comunidad quienes ayudaron a identificar 23 plantas medicinales de uso comunitario. Los habitantes de la comunidad de Sahuangal, en especial los abuelos y las mujeres mayores del hogar cuentan con un amplio conocimiento sobre plantas a las cuales les atribuyen propiedades curativas mismas que son empleadas para tratar diferentes padecimientos o enfermedades.
\end{abstract}

Palabras clave: Saberes ancestrales; Plantas medicinales; Conocimiento ancestral; Alternativa natural

\begin{abstract}
One of the factors of great importance for the survival and perpetuation of humanity has been the knowledge and use of medicinal plants as herbal medicines. In the present case study, the ancestral knowledge of the botanical resources of the Rural parish of Sahuangal, located northwest of Pichincha, Ecuador, was identified. The information collection was carried out through technical visits, participatory workshops, interviews and surveys with the inhabitants of this community who helped identify 23 medicinal plants for community use. The inhabitants of the Sahuangal community, especially the grandparents and elderly women of the household, have extensive knowledge about plants to which they attribute healing properties that are used to treat different ailments or diseases.
\end{abstract}

Key words: Ancestral knowledge; Medicinal plants; Ancestral knowledge; Natural alternative
LS: Economista. Experiencia como docente, Iniciación y Planificación de Proyectos, CSU Formación Integral en Entornos de aprendizaje de Educación Superior, Programa de Formación Docente sobre el Modelo ITSCO, CSU Actualización de Competencias Docentes Orientadas a la Mejora de la Calidad Educativa. Instituto Superior Tecnológico Cordillera, Ecuador. 
LS: Economista. Experiencia como docente, Iniciación y Planificación de Proyectos, CSU Formación Integral en Entornos de aprendizaje de Educación Superior, Programa de Formación Docente sobre el Modelo ITSCO, CSU Actualización de Competencias Docentes Orientadas a la Mejora de la Calidad Educativa. Instituto Superior Tecnológico Cordillera, Ecuador.

\section{RESUMO}

Um dos fatores de grande importância para a sobrevivência e perpetuação da humanidade tem sido o conhecimento e a utilização das plantas medicinais como fitoterápicos. No presente estudo de caso, foi identificado o conhecimento ancestral dos recursos botânicos da freguesia Rural de Sahuangal, localizada a noroeste de Pichincha, Equador. A coleta de informações foi realizada por meio de visitas técnicas, oficinas participativas, entrevistas e pesquisas com moradores dessa comunidade que ajudaram a identificar 23 plantas medicinais para uso comunitário. Os habitantes da comunidade Sahuangal, especialmente os avós e as mulheres idosas da casa, têm amplo conhecimento sobre as plantas às quais atribuem propriedades curativas que são usadas para tratar diferentes doenças ou enfermidades.

Palavras-chave: Conhecimento ancestral; Plantas medicinais; Conhecimento ancestral; Alternativa natural

\section{INTRODUCCIÓN}

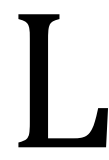
os saberes ancestrales son todos aquellos conocimientos que poseen los pueblos y comunidades indígenas, transmitidos principalmente de manera oral de generación a generación, de padres a hijos (transmisión vertical) o a través de personas mayores o "sabias" (transmisión oblicua), en el marco de las dinámicas de la convivencia comunitaria que caracterizan a nuestros pueblos indígenas (1).

Estos saberes ancestrales abarcan una gran variedad de aspectos del conocimiento y la técnica que va desde el lenguaje hasta la gastronomía, desde las matemáticas hasta la artesanía, pasando por la medicina, la construcción, la silvicultura, las técnicas de conservación del ambiente y microclimas, la producción y alimentación, la agricultura y el riego, el transporte y la comunicación (2).

Los conocimientos tradicionales de las comunidades indígenas y locales están asociados al uso y aplicación de las plantas $\mathrm{y}$ han jugado un rol importante en la economía mundial (3). La producción agrícola y la utilización de plantas como alternativa natural para el tratamiento de enfermedades se han convertido en un eje de gran importancia para el desarrollo económico de las comunidades rurales y su aporte al desarrollo sostenible es relevante.

El reconocimiento de los pueblos indígenas como autores y gestores activos de este conocimiento, en vez de considerarlos como simples fuentes de información, está tomando fuerza en una economía basada en la conservación y aprovechamiento de los recursos renovables. El hecho de que el $65 \%$ de la trasmisión del conocimiento tradicional sea todavía oral, ejemplifica la importancia que tiene la preservación cultural de los pueblos indígenas (4).

En la actualidad, la perdida de los saberes y conocimientos ancestrales es una realidad a nivel mundial y especialmente lo referente al uso de plantas medicinales (5). Entre sus causas se tiene varios factores entre los que podemos mencionar la globalización, la cual ha generado rápidos cambios ambientales, socioeconómicos y culturales y los procesos migratorios de las 
zonas rurales hacia las zonas urbanas, lo que supone un salto generacional el mismo que hace que se pierda la comunicación entre padres e hijos y por lo tanto no haya transmisión del conocimiento del saber ancestral (6).

La Declaración de la UNESCO sobre la Diversidad Cultural (7), establece que los saberes ancestrales son un patrimonio de interés no solo para las comunidades sino para toda la humanidad, el conocimiento transmitido por vía oral permite conservar la biodiversidad existente y por esos motivos debe ser protegida, promovida y consolidada en beneficio de las generaciones futuras.

A nivel mundial el conocimiento local e indígena está experimentando grandes cambios en lo que respecta a cultura, economía y al ambiente, lo que contribuye a debilitar su base de conocimientos locales y amenaza la conservación de esa diversidad biológica (8).

El conocimiento indígena comprende creencias y prácticas que se han ido desarrollando longitudinalmente en relación con su entorno natural; estos conocimientos son dinámicos y coevolucionan junto con los cambios sociales y ecológicos, en la actualidad estos saberes $\mathrm{y}$ biodiversidad se han ido perdiendo notoriamente a nivel mundial (9).

En países como el Ecuador, los saltos generacionales que han ocasionado las oleadas migratorias han aumentado la pérdida de saberes y conocimientos ancestrales provocando que el patrimonio cultural intangible no se preserve de generación en generación (10).

Las familias inmigrantes experimentan profundas transformaciones que determinan cambios en los espacios de socialización de saberes, aumentando la probabilidad de que no se transmitan dichos conocimientos (11). "Las manifestaciones culturales como la lengua, las tradiciones, el territorio, la organización social y los conocimientos sobre la naturaleza y el universo siguen siendo parte esencial de la humanidad" (12).

En este ámbito, resulta notable el esfuerzo que se ha hecho en el Ecuador donde, en el marco del proyecto histórico del "Plan del Buen Vivir 2013-2017" se fomenta el rescate, la preservación y la divulgación de los conocimientos ancestrales (13).

Las plantas constituyen un recurso natural que tiene gran importancia en investigación y desarrollo de nuevos principios activos y medicinales, según el Catálogo de plantas Vasculares elaborado en colaboración con varias instituciones nacionales (del Jardín Botánico de Missouri, el Herbario QCA y la Pontificia Universidad Católica del Ecuador), Ecuador posee alrededor de 18500 especies de plantas vasculares, cerca de 5700 especies endémicas y es el quinto país más diverso del continente americano (14).

Las prácticas ancestrales relacionadas con los usos de la biodiversidad aplicadas a la medicina son de suma importancia; pues, estas prácticas integran la salud humana, el cuidado del medio ambiente y la relación espiritual entre el hombre y la naturaleza. (15), en la actualidad la parte espiritual ha sido olvidada, el hombre se ha desconectado de la naturaleza y con el aparecimiento de nuevas enfermedades se hace necesario rescatar los saberes que reconectan al ser humano con el ámbito natural y espiritual de su entorno (16).

Para Garzón, nutricionista de la Universidad Nacional de Colombia, es importante recuperar la memoria ancestral especialmente lo relacionado con las plantas medicinales y las tradiciones ya que 
constituyen recursos que con el tiempo pueden perderse (17). Además, se debe tomar en cuenta los esfuerzos que se están realizando para el rescate de la soberanía, la seguridad alimentaria y nutricional (18).

La comunidad rural de Sahuangal está ubicada en la Parroquia de Pacto del Distrito Metropolitano de Quito, parroquia Pichincha-Ecuador. Es una zona netamente agrícola y ganadera cuya producción básicamente depende de la leche, caña de azúcar, pitahaya, cacao, café, guabas, cítricos, maíz, maní, plátano y yuca (19).

Su situación geográfica y climática le permiten disponer de una variedad de productos, ubicada en la zona ecuatorial denominada así porque la atraviesa la Línea Equinoccial, está ubicada entre las dos Cordilleras de los Andes y dentro de la Hoya del Río Guayllabamba. Su privilegiada ubicación le permite poseer un clima tanto de la región sierra como de la costa que es Tropical Megatérmico Húmedo (19). Su temperatura oscila entre 18 a $24 \mathrm{oC}$ y una precipitación anual media de 1.800 a 2.200 cc., (20). La flora característica más marcada dentro de la estructura boscosa es la presencia numerosa de epífitas o plantas aéreas como: bromelias, orquídeas, musgos, líquenes, etc.

La comunidad está ubicada aproximadamente a 42 kilómetros al noroccidente de la Parroquia de Pacto, sus coordenadas geográficas son: Latitud 0025483, longitud 17N07455178 y una altura sobre el nivel del mar de $785 \mathrm{~m}$. Sus límites son: al norte la Provincia de Imbabura y el Río Guayllabamba, y la Población de San José de Magdalena Bajo. Al sur: la Población de Anope y el Río Anope. Al este, está la Población del Chontal (Prov. Imbabura) y al oeste se encuentra la Población de la Unión.
Los inventarios de recursos botánicos en una zona permiten el registro y uso de la biodiversidad de las fincas, huertas $\mathrm{y}$ predios de los habitantes y la preservación de sus prácticas ancestrales. La parroquia de Sahuangal no ha realizado inventarios de plantas de la zona, ni ha determinado el uso de los recursos botánicos para uso medicinal; es así que, el objetivo de la investigación fue documentar y describir una guía para fomentar y preservar los conocimientos locales existentes de los habitantes de la comunidad rural de Sahuangal, parroquia Pacto, provincia de Pichincha.

\section{MATERIALES Y MÉTODOS}

S e trató de una investigación descriptiva que incluyó una muestra de 23 plantas de uso medicinal utilizadas por los habitantes, las mismas que fueron identificadas en tres visitas técnicas a la comunidad en donde la participación de la comunidad fue fundamental para la recopilación de la información. La población de Sahuangal estuvo conformada por alrededor de 80 familias de aproximadamente 4 a 5 miembros aproximadamente. En la comunidad existen alrededor de 256 habitantes de los cuales, 141 son hombres y 115 mujeres. El lugar cuenta con un centro de salud, un retén policial, y una escuela, posee obras de alcantarillado y luz eléctrica (19).

Se obtuvo información del uso de las plantas de forma personal mediante visitas a cada hogar de la comuna Sahuangal. Las entrevistas fueron dirigidas en su mayoría a las mujeres adultas del hogar debido a su amplio conocimiento botánico y agrícola. 
El proceso comenzó con una visita a las autoridades de la comunidad donde a través de un taller participativo se definió el tema del proyecto de investigaciónvinculación de interés de los pobladores como la contraparte. Su objetivo fue la recuperación de los saberes y conocimientos ancestrales del sector para su preservación y posterior transmisión a las futuras generaciones.

Se solicitó la autorización para realizar las actividades planteadas para el efecto, luego en una segunda visita se realizó un taller o grupo focal con los pobladores y autoridades para conocer la realidad del sector en cuanto al nivel de conocimiento de saberes ancestrales; se realizó esto, específicamente, para tener una primera idea de las plantas que conocían y que eran utilizadas como alternativa a la medicina tradicional. En este taller se reconoció pobladores con el conocimiento en la utilidad de esas plantas y la forma en como transmitían esos conocimientos.

En una tercera visita se realizó un recorrido de la comunidad hacia las diferentes fincas, y huertos para recopilar información de las plantas útiles y realizar la respectiva identificación y sus usos por parte de los habitantes con información in situ.
Una vez recopilada esa información se plasmó en una guía detallada para comunicación interna de la comunidad.

\section{RESULTADOS}

$\mathrm{S}$ e recopiló información sobre 23 plantas que los habitantes de la comuna de Sahuangal conocen y utilizan como opción para el tratamiento o como paleativos de ciertos padecimientos leves. Estas platas pertenecen a diferentes familias siendo la Lamiaceae y la Malvaceae con tres especies cada una las de mayor representación en el estudio.

El resto de familias botánicas fueron representadas con una especie cada una. Entre las partes que se utilizan de las plantas mencionan que lo más utilizado es las hojas para infusiones y sus semillas como aderezos.

Durante la fase de recopilación de la información los informantes manifestaron que las personas más jóvenes de la comunidad en su gran mayoría desconocen la utilidad medicinal de estas plantas y que se está tendiendo a la utilización de la medicina convencional.

Tabla 1. Plantas Identificadas y su forma de uso.

\begin{tabular}{lll}
\hline Nombre común & Familia & Forma de uso/saber ancestral \\
\hline Sahuango & & Se aplica gotas del tallo en la zona afectada (21) \\
Matico & Scrophulariaceae & Infusión (21) \\
Orégano & Lamiaceae & Aderezo, infusión o té (21) \\
Albahaca & Lamiaceae & Aceite, hojas para aderezo, infusión (21) \\
Hoja de mortiño & Ericaceae & Aderezo, infusión o té. (21) \\
\hline
\end{tabular}




\begin{tabular}{|c|c|c|}
\hline Nombre común & Familia & Forma de uso/saber ancestral \\
\hline Yuquilla & Acanthaceae & Tallo subterráneo, aceite, condimento. (21) \\
\hline Sábila & Asphodelaceae & Extracto de la hoja. (21) \\
\hline Cedrón & Verbenaceae & Infusión, aderezo. (21) \\
\hline Menta & Lamiaceae & Infusión o té. (21) \\
\hline Amaranto & Amaranthaceae & Aceites, infusión. (21) \\
\hline Hoja de naranja & Rutaceae & $\begin{array}{l}\text { Zumo, té o infusión de la hoja o macerado de la misma. } \\
(21)\end{array}$ \\
\hline Achiote & Bixaceae & $\begin{array}{l}\text { Hojas, flor y semillas en infusión, corteza del árbol, raíz, } \\
\text { fruto y aceite de semillas. ( } 21 \text { ) }\end{array}$ \\
\hline Llantén menor & Plantaginaceae & Infusión, hoja en ensaladas o sopas. (21) \\
\hline Hoja de cacao & Malvaceae & Polvo, chocolate, pulpa de fruta. (21) \\
\hline Guayaba & Myrtaceae & Infusión de las hojas, jugo de la fruta. (21) \\
\hline Tilo & Malvaceae & Infusión, cápsulas. (21) \\
\hline Ruda & Rutaceae & Infusión. (21) \\
\hline Valeriana & Caprifoliaceae & Gotas, infusión. (21) \\
\hline Guanábana & Annonaceae & Infusión de las hojas, pulpa de la fruta. (21) \\
\hline Paico & Amaranthaceae & Infusión, crema, aceite, condimento, (21) \\
\hline Escansel & Amaranthaceae & $\begin{array}{l}\text { Las hojas aromáticas y ramas pequeñas se usan como té. } \\
\text { (21) }\end{array}$ \\
\hline Malva & Malvaceae & Infusión. (21) \\
\hline Stevia & Asteráceas & Infusión, extracto. (21) \\
\hline
\end{tabular}


Tabla 2. Nombre científico, uso alternativo y contraindicaciones

\begin{tabular}{|c|c|c|}
\hline Nombre científico & Uso alternativo & Contraindicaciones \\
\hline Sahuango & $\begin{array}{l}\text { Problemas digestivos, tratamiento } \\
\text { de picaduras de picaduras de } \\
\text { insectos. (22) }\end{array}$ & $\begin{array}{l}\text { No se conocen interacciones con } \\
\text { otros fármacos ni efectos } \\
\text { secundarios destacables.(24) }\end{array}$ \\
\hline Piper aduncum & $\begin{array}{l}\text { Afecciones urinarias, amigdalitis, } \\
\text { antiinflamatorio antiséptico vaginal, } \\
\text { infecciones urinarias, infecciones } \\
\text { bronquiales, tratamiento de úlceras } \\
\text { digestivas, indigestión, dolor de } \\
\text { estómago, disfunción hepática. (22) }\end{array}$ & $\begin{array}{l}\text { Antecedentes de alérgica, } \\
\text { embarazo. (24) }\end{array}$ \\
\hline Origanum vulgare & $\begin{array}{l}\text { Infecciones urinarias, infecciones } \\
\text { bronquiales, tratamiento del Acné } \\
\text { Asma, sistema digestivo, dolores de } \\
\text { garganta, antiinflamatorio, } \\
\text { antioxidante, antimicrobiano (23) }\end{array}$ & $\begin{array}{l}\text { No recomendable en embarazo, } \\
\text { sobredosificación } \\
\text { producir: agitación, depresión, } \\
\text { somnolencia (25) }\end{array}$ \\
\hline Ocimum basilicum & $\begin{array}{l}\text { Dolor de garganta, calmante de } \\
\text { picaduras, cefalea, tratamiento del } \\
\text { Acné, mejora el sistema inmune, } \\
\text { antimicrobiano, } \\
\text { antiinflamatorio (23) }\end{array}$ & $\begin{array}{l}\text { Dermatitis Gastritis Ulceras } \\
\text { gástricas Enfermedad Crohn, no } \\
\text { recomendable su aceite esencial } \\
\text { en infancia, embarazo o pacientes } \\
\text { con gastritis. (25) }\end{array}$ \\
\hline $\begin{array}{l}\text { Vaccinium } \\
\text { meridionale }\end{array}$ & $\begin{array}{lr}\begin{array}{l}\text { Prevenir el } \\
\text { enfermedades } \\
\text { Alzheimer }\end{array} & \begin{array}{c}\text { desarrollo de } \\
\text { cardiovasculares, }\end{array} \\
\text { antimicrobiano, } \\
\text { hipoglicemia, diabetes (22) }\end{array}$ & $\begin{array}{l}\text { Pacientes con gastritis o úlcera } \\
\text { gastroduodenal, diabetes, puede } \\
\text { producir diarrea. (24) }\end{array}$ \\
\hline Curcuma longa & $\begin{array}{l}\text { Trastornos hepáticos, biliares y en } \\
\text { caso de malas digestiones estimula } \\
\text { la secreción de jugos gástricos y } \\
\text { promueve la secreción biliar. } \\
\text { Enfermedad de Párkinson, } \\
\text { antiinflamatorio, diurético (22) }\end{array}$ & $\begin{array}{l}\text { Embarazo, puede empeorar los } \\
\text { problemas de la vesícula biliar, } \\
\text { retardar la coagulación } \\
\text { sanguínea. (25) }\end{array}$ \\
\hline Aloe vera & $\begin{array}{l}\text { Diabetes, problemas digestivos, } \\
\text { asma, tratamiento para quemaduras } \\
\text { leves, desintoxicante, cicatrizante, } \\
\text { antiviral, antiinflamatorio, efecto } \\
\text { laxante (23) }\end{array}$ & $\begin{array}{l}\text { Lactancia, en la piel y las heridas } \\
\text { abiertas puede producir } \\
\text { quemaduras, irritaciones. (25) }\end{array}$ \\
\hline Aloysia citrodora & $\begin{array}{l}\text { Bronquitis, } \\
\text { antiinflamatoria, } \\
\text { expectorante (22) }\end{array}$ & $\begin{array}{l}\text { Embarazo, lactancia, } \\
\text { enfermedades renales. (24) }\end{array}$ \\
\hline
\end{tabular}




\begin{tabular}{ll}
\hline Nombre científico & Uso alternativo \\
\hline Mentha piperita & Tratamiento respiratorios, \\
& bronquitis, asma, digestión, ayuda a \\
& disminuir las afecciones de la piel en \\
& la lactancia materna, cuidados de la \\
& piel, pérdida de peso y de memoria \\
& $(23)$
\end{tabular}

Amaranthus

hypochondriacus

Citrus aurantium

Bixa orellana

Plantago lanceolata

Theobroma cacao

Psidium guajava

Tilia platyphyllos
Problemas digestivos hemorragias intestinales Trastornos en el periodo menstrual, anticancerígeno, dolores musculares, depresión, mejora el sueño, obesidad, embarazo y lactancia, (22)

Tratamiento respiratorios, sistema inmunitario, antioxidante, cicatrizante, trastornos gástricos (22)

Astringente antiséptico, antibacterial, antioxidante, expectorante, cicatrizante (22)

Faringitis, laringitis, bronquitis,
colitis, diarrea, cuidados de la piel, molestias de garganta (22)

Dolor agudo, estrés, antioxidante, alivia el mal humor, disminuye niveles de colesterol, diurético, cáncer de piel, excelente para el desempeño sexual (22)

Antidiabético, tratamiento digestivo, antidiarreico, antioxidante, pérdida de peso, cuidados de la piel, disminuir el colesterol (22)

Tranquilizante, cólicos, reumatismo, estrés, induce a la sudoración, antioxidante. (22)

\section{Contraindicaciones}

Embarazo. (24)

Simplemente, las personas que estén siguiendo una dieta para perder peso deben tomarlo con moderación por su aporte calórico. (25)

En pacientes con enfermedades cardiovasculares preexistentes. (25)

Embarazos, lactancia. (24)

En embarazo no usar frecuentemente, lactancia, reacciones adversas por contacto, efecto laxante. (25)

No deben tomarlo aquellas personas que presenten alergias a los derivados del cacao, estreñimiento, acné, hipercolesterolemia grave $\mathrm{y}$ dolencias renales. (25)

No recomendado para personas que padezcan colon irritable, embarazadas ni personas con problemas de corazón. (25)

Embarazo, lactancia, las personas con problemas de estómago o del corazón no deberían tomar infusión de tila. (24) 


\begin{tabular}{|c|c|}
\hline Nombre científico & Uso alternativo \\
\hline Ruta graveolens & $\begin{array}{l}\text { Trastornos digestivos, espasmos, } \\
\text { hemorroides, cólicos estomacales, } \\
\text { problemas circulatorios, trastornos } \\
\text { biliares Como uso externo, para } \\
\text { calmar dolores de oído, dolores por } \\
\text { golpes o artritis (23) }\end{array}$ \\
\hline Valeriana officinalis & $\begin{array}{l}\text { Dolor de cabeza o cefalalgia, dolores } \\
\text { musculares, ansiedad, reducen el } \\
\text { nerviosismo y la agitación, facilita la } \\
\text { conciliación del sueño y mejora su } \\
\text { calidad. (22) }\end{array}$ \\
\hline
\end{tabular}

$\begin{array}{ll}\text { Graviola annona } & \text { Antiespasmódica, tratamientos } \\ \text { muricata } & \text { contra el colesterol y la reducción } \\ & \text { de los triglicéridos, ayuda a } \\ & \text { mantener controlada la presión } \\ & \text { arterial, disminución del insomnio, } \\ & \text { mejorar el sistema inmune, } \\ & \text { previene la anemia, retrasa el } \\ & \text { envejecimiento. (22) }\end{array}$

Dysphania ambrosioides

Aerva sanguinolenta

Malva sylvestris

Stevia rebaudiana
Traumatismo, sinusitis, bronquitis, enfermedades antihelmíntico, antiinflamatorio, parasitarias, analgésico, antiácidos, antiséptico. (22)

Enfermedades pulmonares, anginas, neumonía, enfermedades de los pulmones, resfriados, catarro, dolor de pecho, enfermedad renal, infecciones, infecciones de la vejiga, dolores de cabeza, trastornos del hígado y la depresión. (22)

Tratamientos de enfermedades de las vías respiratorias, descongestión nasal, amigdalitis y laringitis, antiinflamatorias, laxantes, cicatrizantes, calmantes, digestivas, llagas y picaduras de insectos. (23)

Diurético, antiácido, fatiga, trastornos de ansiedad, tratamiento de quemaduras, heridas, edulcorantes artificiales, se utiliza como cicatrizante y antiséptico, tiene propiedades antibióticas. (22)

\section{Contraindicaciones}

Posibilidad de inducir el aborto, desde la medicina antigua ya se mencionaba al té o aceite de ruda como potente abortivo. (25)

Personas con enfermedades psiquiátricas con epilepsia o esquizofrenia No se debe combinar con medicamentos contra la depresión, antiepilépticos o que afectan al sistema nervioso central. (25)

Es prudente evitar su consumo cuando se ha diagnosticado la enfermedad de Parkinson. (25)

Mujeres embarazadas, madres lactantes y niños hasta 3 años o personas con enfermedades hepáticas, renales y auditivas. (25)

No se ha encontrado contraindicaciones. (24)

Se recomienda no abusar de las dosis, para evitar un posible efecto irritante sobre las mucosas gástricas. (25)

Puede provocar molestias estomacales, puede resultar alérgica en ciertas personas. (25) 
En la zona además se han identificado otras especies de plantas medicinales de acuerdo a la literatura revisada que a continuación las nombraremos como parte del conocimiento ancestral de la comunidad: frutipán (Artocarpus altilis), espíritu santo, escancel (Aerba sanguinolenta), geranio, floripondio o guanto (Brugmansia arbórea), Hierba luisa (Aloysia citrodora), Menta (Mentha piperita), orégano (Origanum vulgare), ruda (Ruta graveolens), toronjil (Melissa officinalis), Camacho (Ortalis vetula), pambil (Arecaceae), balsa (Ochroma lagopus), chonta (Bactris gasipaes), helecho (Pteridium aquilinum). (19).

\section{DISCUSIÓN}

$\mathrm{L}$ os habitantes de la comunidad de Sahuangal, en especial los abuelos y las mujeres mayores del hogar cuentan con un amplio conocimiento sobre plantas a las cuales les atribuyen propiedades curativas mismas que son empleadas para tratar diferentes padecimientos o enfermedades. $\mathrm{Y}$ este conocimiento se evidenció en la descripción de las 23 plantas identificadas en este estudio

Lamentablemente los jóvenes de la comunidad, no están adquiriendo este conocimiento como lo hicieron las generaciones de sus padres, abuelos y demás antepasados, por ese motivo, ven con beneplácito la creación de una guía informativa sobre el uso de estas plantas propias de la región para poder difundirla internamente.

Según Ríos, De la Cruz y Mora (26), el Ecuador posee una gran biodiversidad, la cual resulta muy atractiva para la industria farmacéutica puesto que utiliza estos saberes ancestrales relacionados con el mundo vegetal como una fuente de materia prima útil para la medicina occidental.

Para Rincón y González (27) es importante realizar inventarios de plantas útiles porque al recolectar información sobre las características, tipos, utilización y utilidad de las mismas para el tratamiento de enfermedades es posible poner esta información al alcance de todos especialmente de personal técnico o científico eliminando el problema de falta de información sistemática y confiable.

La educación ambiental y la conservación de los conocimientos etnobotánicos son parte de los recursos naturales de los pueblos y comunidades por ello es necesario desarrollar actividades que generen una participación activa y promuevan una cultura ambiental comunitaria en las nuevas generaciones que evite que estos vayan desapareciendo por factores asociados a la globalización. (28).

La medicina "complementaria" o "alternativa" refiere al variado número de prácticas de atención de salud que no forman parte de la tradición del país y que, por distintas circunstancias, no han sido incluidas al sistema sanitario de una nación. Uno de los factores de gran importancia para la supervivencia y perpetuación de la humanidad ha sido el conocimiento y uso de plantas medicinales como medicamentos herbarios (29).

En efecto, actualmente podemos apreciar que el conocimiento y aprovechamiento de plantas medicinales, aún se encuentra arraigado en las costumbres y sistemas tradicionales de salud de diversas culturas y comunidades (entre estas, campesinas e indígenas), en las que, las plantas representan una de las principales opciones para prevenir y dar 
tratamiento a una enfermedad (30). Todavía se requiere profundizar en el tema de estudio de biomoléculas activas en recursos naturales para la prevención, diagnóstico y tratamiento satisfactorio de enfermedades.

Según Bravo (31) en lo que se refiere a la tradición de conocimientos estos no solo son aquellos aprendidos personalmente, sino que también engloban a aquellos que se trasmiten y estos últimos forman parte de nuestros saberes.

El conocimiento de la medicina tradicional se hereda y representa la identidad cultural de los pueblos y comunas, este es transmitido a través de generaciones, de abuelos a padres y padre a hijos manteniendo así ese conocimiento de la utilidad medicinal de las plantas. (32).

Esa transmisión de conocimientos ancestrales se ve amenazada por los cambios sociales, económicos y culturales derivados de la modernización, la globalización y las migraciones (30).

\section{CONCLUSIONES}

$\mathrm{E}$ I número de plantas recopiladas en esta investigación fue de 23, tomando en consideración que son las más utilizadas por los pobladores con usos medicinales, las mismas que fueron documentadas por su nombre común, familia a la que pertenece, forma de uso (saber ancestral), nombre científico, uso alternativo y contraindicaciones, esa información se plasmó en una guía que será de utilidad para preservar y transmitir esos conocimientos a las futuras generaciones de la comunidad de Sahuangal.

Los estudios de biodiversidad de los pueblos y comunidades rurales sirven para describir la riqueza etnobotánica de la zona además permiten a la población en general reflexionar sobre la importancia que tienen los conocimientos y saberes ancestrales como patrimonio intangible de la sociedad.

Los adultos mayores son quienes realizan la trasferencia de conocimiento a las demás generaciones por lo que es importante concientizar a la población sobre la preservación de estos conocimientos para su aprovechamiento por futuras generaciones.

En la descripción de las plantas los habitantes manifestaron un conocimiento de cada planta al identificar físicamente a cada una explicando las propiedades terapéuticas y medicinales, además la forma de consumo y las partes que utilizan de cada una de ellas.

La principal forma de transmisión de esos saberes y conocimientos es de forma oral, lo que se da generalmente dentro del núcleo familiar y al compartirlo entre los habitantes de la comuna.

Además, por esa forma de trasmisión de esos saberes y conocimientos sobre las plantas, se evidenció el riesgo de pérdida de los mismos debido a que los jóvenes de la comunidad no los están adquiriendo, y entre las principales causas de esa posible pérdida del conocimiento estarían los cambios y diferencias generacionales y el desarrollo social y tecnológico fenómenos propios de la globalización que repercuten en los estilos de vida cotidianos.

\section{REFERENCIAS BIBLIOGRÁFICAS}

1. Reyes-García V, Broesch J, Calvet-Mir L, Fuentes-Peláez N, McDade T, Parsa S, Martínez-Rodríguez M. Cultural transmission of ethnobotanical knowledge and skills: an empirical analysis from an 
Amerindian society [Internet]. 2009 [citado 23 nov 2020]. Disponible en: https://doi.org/10.1016/j.evolhumbehav. 2009.02.001

2. Gutiérrez M. El fortalecimiento de los Saberes Ancestrales como un fin de la Educación Superior [Internet]. 2018 [citado 25 nov 2020]. Disponible en: https://www.upec.edu.ec/images/stories/ LOTAIP/2018/JUNIO/ANEXOS/M-1INFORMATIVO.pdf

3. Caillaux J, Ruiz M. La protección jurídica de los conocimientos tradicionales y sus desafíos [Internet]. 2004 [citado 15 nov 2020]. Disponible en: https://repositorio.promperu.gob.pe/han dle/123456789/1431

4. Bajak A. Manejo de colecciones etnobiológicas para descubrimiento $\mathrm{y}$ desarrollo farmacéutico en América Latina [Internet]. 2014 [citado 24 nov 2020]. Disponible en: http://latinamericanscience.org/spanish/ 2014/01/manejo-de-coleccionesetnobiologicas-para-descubrimiento-ydesarrollo-farmaceutico-enamerica-latina/ 5. Benz B, Cevallos J, Santana F, Rosales J, Graf S. Pérdida del conocimiento acerca del uso de la plantas en la reserva de la biosfera sierra de manantlan, México [Internet]. 2014 [citado 26 nov 2020]. Disponible en: https://doi.org/10.1007/BF02907821

6. Herrera G, Moncayo I, Escobar A. Perfil Migratorio del Ecuador 2011 [Internet]. 2012 [citado 21 nov 2020]. Disponible en: https://publications.iom.int/system/files/ pdf/perfil_migratorio_del_ecuador2011.pd $\mathrm{f}$

7. UNESCO. Declaración Universal de la UNESCO sobre la Diversidad Cultural [Internet]. 2001 [citado 12 nov 2020]. Disponible en:

http://portal.unesco.org/es/ev.phpURL_ID $=13179 \& U R L \_D O=D O \_T O P I C \& U R L$ _SECTION=201.html

8. Aswani S, Lemahieu A, Sauer, W. Global trends of local ecological knowledge and future [Internet]. 2018 [citado 12 dic 2020]. Disponible en: https://doi.org/10.1371/journal.pone.019 5440

9. Berkes F, Colding J, Folke C. Redescubrimiento del conocimiento ecológico tradicional como gestión adaptativa [Internet]. 2000 [citado 17 dic 2020]. Disponible en: https://esajournals.onlinelibrary.wiley.co m/doi/full/10.1890/1051-

0761\%282000\%29010\%5B1251\%3AROT EKA\%5D2.0.CO\%3B2

10. Vertovec S. Migrant transnationalism and modes of transformation. Obtenido de International Migration [Internet]. 2006 [citado 19 dic 2020]. Disponible en: https://esajournals.onlinelibrary.wiley.co m/doi/full/10.1890/1051-

0761\%282000\%29010\%5B1251\%3AROT EKA\%5D2.0.CO\%3B2

11. Yumisaca Jiménez $S$. Erosión de conocimientos ancestrales de los productores de papa en cinco comunidades de la UCASAI, parroquia San Juan, cantón Riobamba, provincia de Chimborazo [Internet]. 2013 [citado 21 dic 2020]. Disponible en: http://dspace.ups.edu.ec/handle/123456 789/6406

12. Nodos de Biodiversidad. ¿Por qué es importante rescatar los saberes ancestrales? [Internet]. 2018 [citado 30 nov 2020]. Disponible en: http://conexionbio.jbb.gov.co/importancia -de-rescatar-saberes-ancestrales/

13. SEMPLADES. Plan Nacional de Desarrollo [Internet]. 2017 [citado 28 nov 2020] Disponible en: https://www.planificacion.gob.ec/wpcontent/uploads/downloads/2017/10/PN BV-26-OCT-FINAL_0K.compressed1.pdf

14. Jorgensen $P$, y León S. Catalogue of the vascular plants of Ecuador [Internet]. 1999 [citado 28 nov 2020]. Disponible en: http://www.mobot.org/mobot/research/ ecuador/welcomesp.shtml

15. Becerra Palma ME. Costumbres $y$ prácticas que utilizan los agentes de la medicina ancestral y su relación en la salud de los moradores, en la parroquia Chinga recinto Chigüe de la provincia de 
Esmeraldas del año 2014 [Internet]. 2014 [citado 18 nov 2020]. Disponible en: https://repositorio.pucese.edu.ec/handle/ 123456789/304

16. Ocampo J, Guerrero I, Noreña V, Valencia D. Construcción de la memoria colectiva de los pobladores de Nueva Jerusalén sector La Paz conocedores de saberes populares medicinales no farmacéuticos [Internet]. 2019 [citado 19 dic 2020] Disponible en: https://hdl.handle.net/10656/11029

17. Nodos de Biodiversidad. ¿Por qué es importante rescatar los saberes ancestrales? [Internet]. 2018 [citado 19 dic 2020]. Disponible en: http://conexionbio.jbb.gov.co/importancia -de-rescatar-saberes-ancestrales/

18. De la Roca P. Seguridad Alimentaria, Soberanía Alimentaria y Pueblos Indígenas. Estudios Interétnicos [Internet]. 2014 [citado 23 nov 2020] Disponible en: http://c4.usac.edu.gt/revindex/articulos/ editor9-

r331_pi44_pfi61_ra100REVISTAESTUDIOS INTERETNICOS-25.44-61.pdf

19. Proaño G. Estudio de prefactibilidad para la implementación de alternativas de desarrollo turístico en la Comunidad de Sahuangal, ubicada en la parroquia de Pacto, cantón Quito, provincia de Pichincha [Internet]. 2013 [citado 19 dic 2020] Disponible en: http://repositorio.ute.edu.ec/handle/123 $456789 / 13412$

20. Cañadas L. El Mapa Bioclimatico y Ecológico del Ecuador [Internet]. 2010 [citado 22 dic 2020] Disponible en: http://bibliotecas.espoch.edu.ec/cgibin/koha/opac-

detail.pl?biblionumber $=20615 \&$ shelfbrow se_itemnumber $=32344$

21. De la Torre L, Navarrete $H$, Muriel $P$, Macía M, Balslev H. Enciclopedia de las Plantas Útiles del Ecuador [Internet]. 2008 [citado 02 dic 2020]. Disponible en: https://www.academia.edu/30089423/En ciclopedia_de_Plantas_U_tiles_del_Ecuador 22. Sánchez D. Las Plantas y sus Utilidades Medicinales [Internet]. 2005 [citado 22 dic
2020].

Disponible

en: https://es.scribd.com/document/4338036 18/libro-de-plantas-medicinales-2005-pdf 23. Pineda J. Plantas Medicinales [Internet]. 2013 [citado 22 dic 2020]. Disponible en: https://encolombia.com/saludestetica/medicinaalternativa/caseros/plantas-medicinales/ 24. Castillero O. Las 50 mejores plantas medicinales, sus efectos y contraindicaciones [Internet]. 2014 [citado 22 dic 2020]. Disponible en: https://psicologiaymente.com/salud/plan tas-medicinales

25. Ministerio de la Protección Social. Vademécum Colombiano de Plantas Medicinales [Internet]. 2008 [citado 22 dic 2020]. Disponible en: https://www.minsalud.gov.co/sites/rid/Li sts/BibliotecaDigital/RIDE/VS/PP/SA/vad emecum-colombiano-plantas-

medicinales.pdf

26. Ríos $M$, De la Cruz $R$, Mora $A$. Conocimiento tradicional y plantas útiles del Ecuador: saberes y prácticas" [Internet]. 2008 [citado 15 dic 2020]. Disponible en: https://isbn.cloud/9789978227220/cono cimiento-tradicional-y-plantas-utiles-delecuador-un-sendero-participativo-desaberes-y-prac/

27. Rincón F, González L. Importancia de los Sistemas de Documentación en el Manejo de los Recursos Fitogenéticos [Internet]. 1991 [citado 16 nov 2020]. Disponible en: https://dialnet.unirioja.es/servlet/articulo ?codigo $=5532970$

28. Boeri P, Piñuel L, Zubillaga F, Dalzotto D, Sharry S. "Revaluing Ancestral Knowledge": an extension experience with our senior citizens [Internet]. 2017 [citado 18 nov 2020] Disponible en: http://revista.agro.unlp.edu.ar/index.php/ revagro/article/view/1471/1182

29. Hurtado V. Conocimiento etnobotánico de plantas medicinales en el municipio de Risaralda, Caldas: veredas Banderas y Betania. Revista Cultura y Droga, 144-175 
[Internet]. 2020 [citado 19 dic 2020] Disponible

en: https://doi.org/10.17151/culdr.2020.25.3 0.7

30. Zuluaga G. Bioprospección y pueblos indígenas. Reflexiones para la conservación de la diversidad biológica y cultural [Internet]. 2001 [citado 23 nov 2020]. Disponible en: https://core.ac.uk/download/pdf/480353 99.pdf

31. Bravo Díaz A. Cuando los Saberes se hacen audibles, la transmisión del conocimiento en el Ecuador del Siglo XXI [Internet]. 2013 [citado 27 nov 2020] Disponible http://200.41.82.22/bitstream/10469/57 15/2/TFLACSO-2013AEBD.pdf

32. Bynoe M. "Medicina tradicional y la transmisión de conocimiento ancestral en la comunidad afroboliviana de Tocaña [Internet]. 2018 [citado 16 dic 2020] Disponible en: SIT DIGITAL COLLECTIONS: https://digitalcollections.sit.edu/isp_collec tion/2773/

Conflicto de intereses. El autor declara que no existe conflicto de intereses para la publicación del presente artículo.

Financiamiento. Autofinanciamiento

Agradecimiento. No declaran 